\title{
Towards Specifying Multimodal Collaborative User Interfaces: A Comparison of Collaboration Notations
}

\author{
Frédéric Jourde $^{1}$, Yann Laurillau ${ }^{1}$, Alberto Moran ${ }^{2}$, Laurence Nigay ${ }^{1}$ \\ ${ }^{1}$ Grenoble Informatics Laboratory (LIG) \\ 38042, Grenoble, France \\ \{fjourde,laurilla,nigay\}@imag.fr \\ ${ }^{2}$ Facultad de Ciencias, UABC, \\ Ensenada, Mexico \\ alberto_moran@uabc.mx
}

\begin{abstract}
Interactive systems including multiple interaction devices and surfaces for supporting the collaboration of a group of co-located users are increasingly common in various domains. Nevertheless few collaborative and multimodal interface specification notations are proposed. As a first step towards a notation for specifying a design solution prior to its software design and development, we adopt an empirical approach. We applied and compared four existing notations for collaborative systems by considering a case study, namely, a system for supporting informal co-located collaboration in hospital work. This paper reports the conclusions from this empirical comparison.
\end{abstract}

Keywords: CSCW, multimodality, multi-devices, specification notation.

\section{Introduction}

The multimodal domain, including multi-surface and multi-device areas, has expanded rapidly. Significant achievements have been made in terms of both modalities and multimodal applications especially for Computer-Supported Cooperative Work such as co-located collaboration in a smart room. Real collaborative multimodal systems are now built in various domains [12] including the medical one [10]. Moving away from research prototypes, we now observe the need for specifying such interactive systems especially in the context of industrial projects. In this article, we address this problem of specification of multimodal collaborative User Interfaces (UI).

Specifying user interfaces is a well-established discipline and various notations have been proposed for specifying the tasks, the dialog elements, the sequences of interaction, concrete UI elements, dynamics of group behavior and so on. Such a variety of notations both in terms of their descriptive qualities, their syntactic structures and the amount of support that they offer according to the development phases has already been highlighted ten years ago in [5]. In [9], the review of notations for interaction design underlines that the most common interaction representational needs are covered by four models: task, domain, abstract and concrete UI. Many of these notations are dedicated to single user WIMP interfaces and we are interested in studying the proposed extensions of these notations and more 
recent notations dedicated to collaborative and multimodal UI. To do so, our approach for studying existing notations for specifying multimodal collaborative UI is in the first instance empirical: we start from existing collaboration notations and we apply them for specifying a case study: a system for supporting informal co-located collaboration in hospital work.

\section{Empirical Comparison of Collaboration Notations}

The relationships between collaborative and multimodal interaction open a vast world of possibilities that has not been systemically explored in terms of specification notations. We aim at going further than considering multimodal aspects such as the CARE properties [2] for the concrete UI and collaborative aspects for the abstract UI. In our empirical comparison, we first focus on existing notations for specifying collaborative UI. Since we are interested in also modeling multimodal interaction, while studying collaborative UI specification notations, we also examine the power of expression of the notations for specifying concrete UI. A complementary approach to ours would be to start from multimodal UI specification notations.

\subsection{Scope of the Comparative Study}

In [1], three dimensions for evaluating an interaction model are described: descriptive power (i.e., ability to describe a UI), evaluative power (i.e., ability to help assess multiple design alternatives) and generative power (i.e., ability to help designers create new designs). As a starting point for our comparative study, we are focusing on the descriptive power of UI specification notations. Their impact on the design including their evaluative and generative powers will be studied afterwards. Moreover, our study does not aim at evaluating the selected notations that can be studied in light of the criteria identified in [5] and of the notational dimensions of the framework "cognitive dimensions of notations" [4]. Since the selected notations differ in their descriptive qualities, some focusing on collaborative tasks while others on the users' roles and on collaborative situations, our goal is to assess their complementary aspects and their projected ability to specify a multimodal collaborative user interface.

\subsection{Rational for the Selected Notations}

Our review of existing collaboration notations highlights the fact that the notations such as CTT [11], CUA [4], GTA [15] and MABTA [8], mainly focus on both individual and collaborative tasks. Such notations aim at accommodating several aspects of collaborative work situations into a task specification and thus extend task specification with contextual information. Some notations also focus on other aspects than individual and collaborative tasks such as TKS [7] which focuses on users' knowledge involved in task behavior and UML-G [14] on modeling shared data. 
Amongst the existing notations, a first way for selecting the ones to be applied to our case study would be based on the syntactic structure of the notations (i.e., graphical, tabular, textual approaches) as in [6]. This solution was not satisfying since most of the notations imply several types of representations. Moreover since our study focuses on the specification of a collaborative user interface, we did not consider the notations that are not dedicated to interaction tasks and system behaviors, although they may be complementary to the other notations. We therefore exclude TKS. Moreover although CUA is focusing on individual and collaborative tasks in the context of scenarios, its main focus is on modeling the tasks for the needs of groupware evaluation. As a conclusion, we selected four notations, CTT, GTA, MABTA and UML-G that involve different background disciplines. UML-G is an extension of a standard in Software Engineering. CTT is a well established notation for task analysis in Human-Computer Interaction, while GTA and MABTA aims at extending task analysis with elements from Social Sciences (social psychology, sociology) in order to capture key elements of the nature of groupworking.

\section{Specifications Based on the Selected Notations}

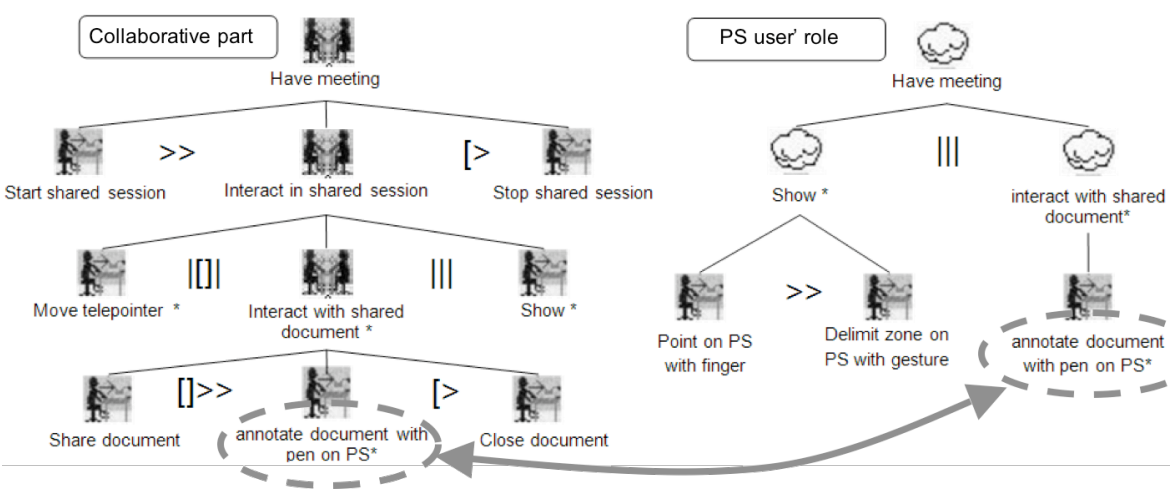

Fig. 1. CTT collaborative and public display (PS user's role) individual task trees.

We apply the four selected notations to specify a collaborative multi-surface [10] that provides a support for informal co-located collaboration by allowing multiple users to study medical documents. As highlighted by the field study described in [10], hospital medical workers including physicians and medical interns are very mobile and need to opportunistically and informally establish co-located collaboration while focusing on a particular patient. Using the system, two physicians can share extracts from a patient medical displayed both on the large screen (i.e., Public Screen) and on the PDA screen (i.e., Private Screen): (i) on the public screen, physicians can only annotate the medical information using a virtual pen ; (ii) on the PDA, a physician can initiate a shared session, select documents to share, edit documents and stop a session. For illustrative purpose, we partly present the CTT specification of our case study 
while the complete specifications along the four notations are available at [16]. Applying CTT, collaborative activities are described at a high-level of abstraction through a collaborative task tree; individual task trees (one per role) describe concrete tasks. A collaborative task tree contains collaborative tasks and high-level individual tasks, related to one of the multiple individual task trees. Figure 1 shows the collaborative task tree for our case study and one individual task tree associated with the public screen user's role. The CTT notation includes five types of tasks: system, mental, abstract, individual (user) and collaborative tasks. A collaborative task is an abstract task that must be composed of individual tasks. Relation operators between tasks are inherited from LOTOS. In particular, Figure 1 highlights the coupling between the PDA and PS (I[]l operator) and the document sharing between users ([]>> operator).

Difficulties or limitations identified by applying CTT include the fact that the links between the tasks of different trees are not explicit (i.e., no role is specified for a task) and are only deduced from the task identifiers. Moreover to specify the modalities of a concrete task, the only means is to use the task identifier (e.g., Annotate document with pen on $P S$ ). In addition, the notation does not provide any means of representing shared objects and of specifying a policy for the sharing. For example, we are able to specify that the telepointer is controlled by the PDA but we are not able to specify that the telepointer can be observed by both roles.

\section{Conclusions from the Comparative Study and Future Work}

About role specification, the four notations explicitly support user's roles specification in working group. While GTA and MABTA advocate a dedicated representation to roles and relationships between users, we describe roles using UML$\mathrm{G}$ with a class diagram and the involved objects for each role. As opposed to UML-G that focuses on the manipulated objects per role, CTT describes the tasks per role in individual task trees (Figure 1).

About group and individual work specification at abstract level, on the one hand, CTT and MABTA advocate a dedicated representation that combines collaborative tasks, and individual tasks that take part directly in the group work coordination, such as start shared session in Figure 1. However, CTT operators refine the MABTA "influence" relation for the case of temporal interdependencies only. As pointed out in [3], in addition to temporal interdependencies related to the activity level, interdependencies are related to the object level and describe the multiple participants' access to the same set of objects. In [13], they define a set of generic mechanics of collaboration as elementary abstract tasks for such coordination issues surrounding how objects are assessed. Such elementary abstract tasks are generic since they are common to a variety of social and organizational work groups. For example one abstract task "Obtain resource" could be part of the CTT and MABTA group work representations.

On the other hand, GTA and UML-G represent together group and individual work. Using GTA, it is possible to annotate each task with the roles and the manipulated objects. As for GTA, with UML-G, group work is implicitly described 
within the class diagram by different roles manipulating the same object. Collaborative activities are further described in the UML-G activity diagram that highlights the relationships between the individual tasks over time.

Individual tasks are described hierarchically in CTT, MABTA and GTA for each role. MABTA refines the work group tasks into sub-tasks while maintaining the columns for describing the roles and adding new individual tasks that are not related to the group work. GTA advocates only one representation for both group and individual works. Links between tasks of different roles can be specified by triggered task and hence corresponds to the "influence" relation of MABTA. As opposed to MABTA and GTA, CTT does not explicitly describe the links between the tasks of different roles. This link is deduced from the group work representations that share tasks with the individual task trees (Figure 1). In contrast to the hierarchical refinement approach of CTT, MABTA and GTA, the activity diagram in UML-G shows individual work and interdependencies with respect to time and roles. Moreover only GTA and UML-G enables us to represent task flows respectively in terms of activity diagram and sequence diagram.

Finally, about group and individual work specification at concrete level, CTT and MABTA advocate the same representation for abstract and concrete tasks. The GTA elementary abstract tasks are described using NUAN which enables a precise description of both users' actions, system feedback and dialogue states. For UML-G, concrete tasks can be described by sequence diagrams along with state-transition diagrams. For each object, the users' actions on it as well as its reactions are described. Nevertheless such a specification would be extremely tedious for a complete user interface.

To conclude, by applying four existing notations for specifying a simple groupware where two users are working on a medical image using a PDA and a public display enables us to identify some complementary aspects in the induced representations as well as some missing aspects. We underline three key issues from this empirical study. Firstly, the distinction between group work and individual work (per role) is useful in a specification for describing at different level of detail (i.e., abstract and concrete) a collaborative user interface from its two facets, the group and the users. However a unified representation of group and individual work enables us to depict interdependencies between users with respect to time and roles. Classical hierarchical representations such as CTT are suitable for individual tasks, while group work representations need to include specific aspects of collaboration such as in MABTA where tasks are decorated with concepts from coordination theory. Secondly, temporal relationships between tasks for describing group work are not sufficient: Temporal interdependencies are at the activity level and interdependencies related to the object level are required for describing the multiple users' access to the same set of objects. UML-G focusing on shared objects can be used for describing such interdependencies. Thirdly, the specification of concrete multimodal interaction as concrete tasks involves extending the selected notations dedicated to WIMP user interfaces. For example, it was not possible to explicitly specify the redundancy (one of the CARE properties of multimodality [2]) of the display (PDA and public display) of our case study. Further studies must be done on the description of tightly coupled multimodal interaction (a concrete multimodal group task corresponding to an abstract group task) and on loosely coupled multimodal interaction (concrete 
multimodal individual tasks corresponding to abstract individual tasks that define a composed abstract group task).

As further work, we plan to experiment on the complementary usage of the studied notations on another case study, namely a collaborative and multimodal military command post. The focus will be on studying the links between the activity (task) and shared resource (object) aspects and on extending the notations in order to depict multimodal interaction. For multimodal interaction, distinguishing abstract/concrete tasks as well as group/individual tasks allows us to identify: (1) tasks that require tightly coupled multimodal interaction when two users are continuously engaged with the accomplishment of physical actions for realizing a concrete group task. (2) Tasks that require loosely coupled multimodal interaction when two users are performing actions along different modalities for realizing two concrete individual tasks that define an abstract group task. For specifying these two types of multimodal group tasks, one of our research avenues is to study extensions of the ICARE notation [2].

\section{References}

1. Beaudouin-Lafon, M.: Designing Interaction, not Interfaces. AVI'00, pp. 15-22, ACM.

2. Bouchet, J., Nigay L., Ganille, T.: ICARE Software Component for Rapidly Developing Multimodal Interfaces. ICMI'04, pp. 251-258, ACM.

3. Ellis, C. A., J. Wainer, J.: A Conceptual Model of Groupware. CSCW'94, pp. 79-8, ACM.

4. Green, T.: Instructions and descriptions: some cognitive aspects of programming and similar activities, AVI'00, pp. 21-28, ACM.

5. Johnson, C.W.: The Namur Principles: Criteria for the Evaluation of User Interface Notations. DSVIS'96, Springer.

6. Johnson, C.W.: The Evaluation of User Interface Design Notations. DSVIS'96, pp. 188206, Springer.

7. Johnson, H., Hyde, J.: Towards Modeling Individual and Collaborative Construction of Jigsaws Using Task Knowledge Structures. TOCHI, 2003, 10 (4), pp. 339-387, ACM.

8. Lim, Y.K.: Task models for groupware and multitasking: Multiple aspect based task analysis (MABTA) for user requirements gathering in highly-contextualized interactive system design. TAMODIA'04, pp. 7-15, ACM.

9. Markopoulos, P. and Marijnissen, P.: UML as a representation for Interaction Design. OZCHI'2000, pp. 240-249.

10. Mejia, D.A., Morán, A.L, and Favela, J., Supporting Informal Co-located Collaboration in Hospital Work. CRIWG'07, pp. 255-270, Springer.

11. Mori, G., Paterno, F.,Santoro, C.: CTTE : Support for Developing and Analyzing Task Models for Interactive System Design. TOSE, 2002, 28 (8), pp.797-813, IEEE Press.

12. Oviatt, S. et al:: Designing the user interface for multimodal speech and gesture applications. HCI, 2000, 15 (4), pp. 263-322, Taylor \& Francis.

13. Pinelle, D., Gutwin, C. Greenberd, S.: Task Analysis for Groupware Usability Evaluation, TOCHI, 2003, 10 (4), pp.281-311, ACM.

14. Rubart, J., Dawabi, P.: Shared data modeling with UML-G. IJCAT, 2004, 19 (3/4), pp. 231-243, Inderscience.

15. Veer, G., Welie, M.: Task Based Groupware Design: Putting Theory into Practice. DIS'00, pp. 326-337, ACM

16. http://iihm.imag.fr/laurillau/four-notations-comparison.pdf 